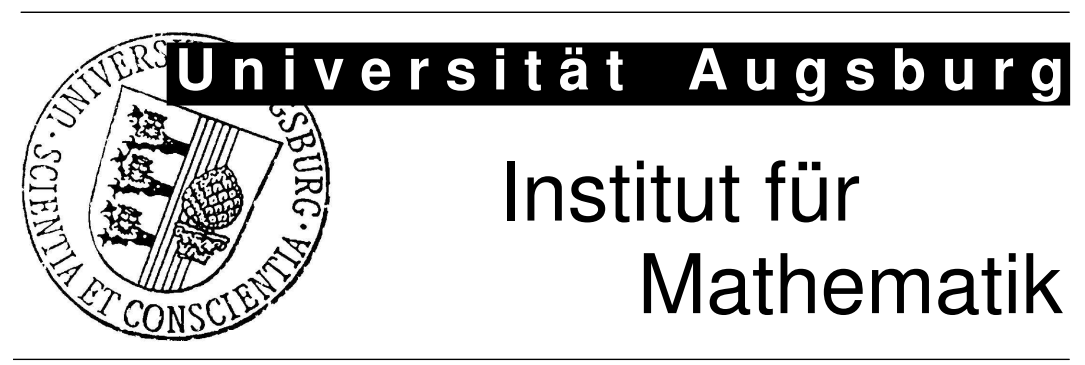

Nguyen Thanh Son, Tatjana Stykel

Model Order Reduction of Parameterized Circuit Equations Based on Interpolation 


\section{Impressum:}

\section{Herausgeber:}

Institut für Mathematik

Universität Augsburg

86135 Augsburg

http://www . math. uni-augsburg.de/de/forschung/preprints.html

\section{ViSdP:}

Tatjana Stykel

Institut für Mathematik

Universität Augsburg

86135 Augsburg

Preprint: Sämtliche Rechte verbleiben den Autoren (C) 2014 


\title{
Model Order Reduction of Parameterized Circuit Equations Based on Interpolation
}

\author{
Nguyen Thanh Son · Tatjana Stykel
}

\begin{abstract}
In this paper, the state-of-the-art interpolation-based model order reduction methods are applied to parameterized circuit equations. We analyze these methods in great details, through which the advantages and disadvantages of each method are illuminated. The presented model reduction methods are then tested on some circuit models.
\end{abstract}

Keywords Parameterized circuit equations - Parametric model order reduction . Interpolation

Mathematics Subject Classification (2000) $\cdot 65 \mathrm{D} 05 \cdot 65 \mathrm{~F} 30 \cdot 93 \mathrm{C} 05 \cdot 94 \mathrm{C}$

\section{Introduction}

Model order reduction is known to be a crucial tool for the simulation of very large integrated circuits and interconnects. This issue attracted the attention of many researchers. It can be seen through numerous published works $[20,22,24,26,28,38$, 39], just to name a few.

This work was done when the first author was with Institute of Mathematics, University of Augsburg. It was supported by the Research Network MoreSim4Nano - Model Reduction for Fast Simulation of new Semiconductor Structures for Nanotechnology and Microsystems Technology, grant 05M10WAC, funded by the German Federal Ministry of Education and Science.

N. T. Son

Department of Mathematics and Informatics, Thai Nguyen University of Science, Quyet Thang Ward, Thai Nguyen City 23000, Vietnam

Tel: +842803609819

E-mail: ntson@tnus.edu.vn

T. Stykel

Institute of Mathematics, University of Augsburg, Universitätsstr. 14, 86159 Augsburg, Germany

Tel.: +49 821-598-2190, Fax: +49 821-598-2193

E-mail: stykel@math.uni-augsburg.de 
Using the modified nodal analysis (MNA), see, e.g., [44], linear RLC circuits can be modeled by a system of differential-algebraic equations (DAEs)

$$
\begin{aligned}
E \dot{x}(t) & =A x(t)+B u(t), \\
y(t) & =B^{T} x(t),
\end{aligned}
$$

where $x(t) \in \mathbb{R}^{N}$ is the state vector containing the node potentials and currents through inductors and voltage sources, $u(t) \in \mathbb{R}^{m}$ is the input vector consisting of the currents of current sources and the voltages of voltage sources, and $y(t) \in \mathbb{R}^{m}$ stands for the output vector. It is the minus of the voltages of current sources and the currents of voltage sources. The system matrices have the form

$$
E=\left[\begin{array}{ccc}
A_{\mathcal{C}} \mathcal{C} A_{\mathcal{C}}^{T} & 0 & 0 \\
0 & \mathcal{L} & 0 \\
0 & 0 & 0
\end{array}\right], A=\left[\begin{array}{ccc}
-A_{\mathcal{R}} \mathcal{G} A_{\mathcal{R}}^{T} & -A_{\mathcal{L}} & -A_{\mathcal{V}} \\
A_{\mathcal{L}}^{T} & 0 & 0 \\
A_{\mathcal{V}}^{T} & 0 & 0
\end{array}\right], B=\left[\begin{array}{cc}
-A_{\mathcal{I}} & 0 \\
0 & 0 \\
0 & -I
\end{array}\right]
$$

where $A_{\mathcal{C}}, A_{\mathcal{L}}, A_{\mathcal{R}}, A_{\mathcal{V}}$ and $A_{\mathcal{I}}$ are the incidence matrices describing the circuit topology, and $\mathcal{G}, \mathcal{L}$ and $\mathcal{C}$ are the conductance, inductance and capacitance matrices, respectively. For practical models, the state space dimension $N$ depends on the number of circuits components and is usually huge. The simulations of such largescale circuit equations are unfeasibly time consuming. The main task of model order reduction is to approximate system (1) by a model of lower dimension which inherits some important physical properties from (1) such as passivity and reciprocity. System (1) is passive if its transfer function $H(s)=B^{T}(s E-A)^{-1} B$ is positive real, i.e., $H(s)$ is analytic in the open right half-plane $\mathbb{C}_{+}$and $H(s)+H(s)^{*} \leq 0$ for all $s \in \mathbb{C}_{+}$. Furthermore, system (1) is reciprocal if $H(s)=S H(s)^{T} S$ for all $s \in \mathbb{C}$, where $S \in \mathbb{R}^{m \times m}$ is a diagonal signature matrix satisfying $S^{2}=I$. Note that if the circuit has neither $V$-loops nor $I$-cutsets and the element matrices $\mathcal{G}, \mathcal{L}$ and $\mathcal{C}$ are symmetric and positive definite, then (1) is passive and reciprocal.

In circuit design and optimization problems, the system matrices in (1) depend, in general, on some parameters that describe the geometric and material properties of circuits. For example, when modeling a transmission line by an equivalent lumped circuit shown in Figure 1, the length of the line and the number of segments may vary. Also, the material used to manufacture the cable will directly affect the circuit components. In this case, the capacitance, conductance and inductance matrices are constructed dependently on all these values, and the circuit equations become

$$
\begin{aligned}
E(p) \dot{x}(t) & =A(p) x(t)+B u(t), \\
y(t) & =B^{T} x(t),
\end{aligned}
$$

where

$$
E(p)=\left[\begin{array}{ccc}
A_{\mathcal{C}} \mathcal{C}(p) A_{\mathcal{C}}^{T} & 0 & 0 \\
0 & \mathcal{L}(p) & 0 \\
0 & 0 & 0
\end{array}\right], \quad A(p)=\left[\begin{array}{ccc}
-A_{\mathcal{R}} \mathcal{G}(p) A_{\mathcal{R}}^{T}-A_{\mathcal{L}}-A_{\mathcal{V}} \\
A_{\mathcal{L}}^{T} & 0 & 0 \\
A_{\mathcal{V}}^{T} & 0 & 0
\end{array}\right]
$$

with a parameter vector $p \in \mathbb{P} \subset \mathbb{R}^{d}$. We assume that the parameter domain $\mathbb{P}$ is connected and the dependencies of system matrices on $p$ are smooth enough to 


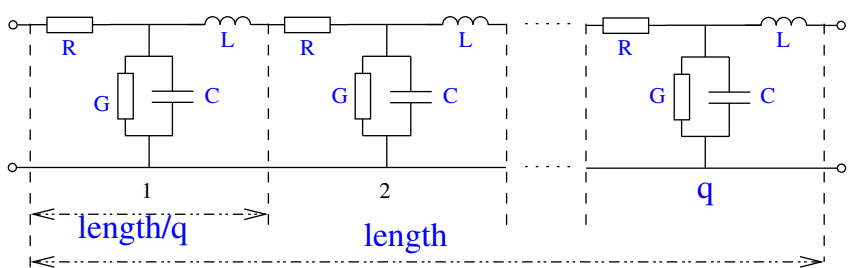

Fig. 1 Lumped-element transmission line

perform later steps. Our task now is to approximate the parameterized system (2) by a reduced-order model

$$
\begin{aligned}
\hat{E}(p) \dot{\hat{x}}(t) & =\hat{A}(p) \hat{x}(t)+\hat{B}(p) u(t), \\
\hat{y}(t) & =\hat{C}(p) \hat{x}(t),
\end{aligned}
$$

where $\hat{E}(p), \hat{A}(p) \in \mathbb{R}^{r \times r}, \hat{B} \in \mathbb{R}^{r \times m}, \hat{C} \in \mathbb{R}^{m \times r}$ and $r \ll N$. It is required that the output $\hat{y}$ of (4), which actually depends on the parameter $p$, approximates the output $y$ of the original system (2), i.e., the approximation error $\hat{y}-y$ is small. In the frequency domain, the approximation error can be measured as $\hat{H}-H$ in an appropriate system norm, where

$$
\begin{aligned}
H(p, s) & =B^{T}(s E(p)-A(p))^{-1} B, \\
\hat{H}(p, s) & =\hat{C}(p)(s \hat{E}(p)-\hat{A}(p))^{-1} \hat{B}(p)
\end{aligned}
$$

are the parameterized transfer functions of (2) and (4), respectively. Note that even if $B$ is parameter-independent, the reduced input and output matrices may depend on $p$.

The problem formulated above has been well established and known as parametric model order reduction (PMOR). The main point here is that the dependence of the original system on parameters should be symbolically preserved or the reduced system should be easily adapted to the parameter changes, so that for each new parameter value, the corresponding reduced-order model can be simulated without reducing the order again. Several approaches have been proposed for the PMOR problem. The authors of $[12,17,21,23,34]$ proposed to match the so-called generalized moments of the transfer function $H(p, s)$ through projecting the original system on (the union of) generalized Krylov subspaces. A closely related interpolatory $\mathcal{H}_{2}$-optimal model reduction method for parameterized systems was considered in $[8,11]$. In $[9,10,40]$, first the balanced truncation method [35] was used to reduce the order of the original system at selected grid points in the parameter domain and then a parameter-dependent reduced transfer function was constructed through interpolating the reduced transfer functions. Henceforth, we refer to this approach as interpolation in the frequency domain. Instead of interpolating the reduced transfer functions, it has been proposed in $[3,5,18,37]$ to interpolate the reduced system matrices to get the reduced-order model on the whole parameter domain. From now on, this approach will be referred to as interpolation in the time domain. Another idea of using interpolation, which has been investigated in $[4,41]$, is the construction of the parameter-dependent projection matrices via the interpolation of the projection subspaces for systems at the grid 
points. Some other PMOR methods can be found in $[14,15,29,30,32,31]$, see also

[13] for a recent survey on state-of-the-art methods in parametric model reduction.

Unfortunately, these PMOR approaches have not been extensively employed for parameterized circuit equations. Besides the moment matching based methods $[17$, $28,33,34$ ] and the reduced basis technique [19], only interpolation in the frequency domain for circuit systems in the scattering form has been considered in [24]. In this paper, the three aforementioned interpolation-based PMOR methods will be investigated for reducing the order of the parameterized MNA system (2), (3). Our goal is twofold. First, we present an extension of these methods to parameterized DAEs and, secondly, we exploit the network structure to make them more efficient for circuit equations. As noted in [43], the standard model reduction methods cannot always be directly applied to DAE systems and their extensions often require a special attention due to algebraic constraints on a solution space.

The remainder of this paper is organized as follows. In Section 2, three interpolation based PMOR methods are reviewed. They are also accompanied by detailed remarks on the practical aspects and application to circuit equations. Section 3 contains some results of numerical experiments demonstrating the properties of the presented model reduction methods. Finally, the conclusion is given in Section 4 .

\section{Model reduction methods for parameterized circuit systems}

Let $p_{0}, \ldots, p_{k} \in \mathbb{P}$ be different parameter vectors selected as interpolation points. At each grid point $p_{i}$, we approximate the so-called local DAE system

$$
\begin{aligned}
E_{j} \dot{x}_{j}(t) & =A_{j} x_{j}(t)+B u(t), \\
y_{j}(t) & =B^{T} x_{j}(t)
\end{aligned}
$$

with $E_{j}=E\left(p_{j}\right)$ and $A_{j}=A\left(p_{j}\right), j=0, \ldots, k$, by a reduced-order model

$$
\begin{aligned}
\hat{E}_{j} \dot{\hat{x}}_{j}(t) & =\hat{A}_{j} \hat{x}_{j}(t)+\hat{B}_{j} u(t), \\
\hat{y}_{j}(t) & =\hat{C}_{j} \hat{x}_{j}(t)
\end{aligned}
$$

using any known projection-based model reduction method. In this case, the system matrices in (6) have the form

$$
\hat{E}_{j}=Z_{j}^{T} E_{j} W_{j}, \quad \hat{A}_{j}=Z_{j}^{T} A_{j} W_{j}, \quad \hat{B}_{j}=Z_{j}^{T} B, \quad \hat{C}_{j}=B^{T} W_{j},
$$

where the projection matrices $Z_{j}, W_{j} \in \mathbb{R}^{N \times r}$ determine, respectively, the subspaces $\mathcal{Z}_{j}$ and $\mathcal{W}_{j}$ of interest. For example, in moment matching approximation and interpolatory model reduction, the columns of $Z_{j}$ and $W_{j}$ form the bases of certain (rational) Krylov subspaces associated with (5), e.g., [6,26]. Since for circuit equations, the preservation of passivity and reciprocity is of great importance, we determine the reduced-order local systems (6) using the PAssivity-preserving Balanced Truncation method for Electrical Circuits (PABTEC) presented in [38,39]. Also, PRIMA and SPRIM algorithms $[27,36]$ can be employed for computing the projection matrices $Z_{j}$ and $W_{j}$.

The reduced-order models (6) can now be used to construct the reduced-order model (4) using an interpolation approach. A general idea of interpolation is to evaluate the unknown data at any points using pre-computed or known data on 
a chosen grid. Applied to the PMOR problem, the known data are the reducedorder systems at $p_{j}$, and one needs to compute the reduced-order model at any parameter $p \in \mathbb{P}$. Such a model can be determined in the frequency domain by its transfer function, in the time domain by its system matrices or by the projection subspaces on which the original system is projected. In this section, these three different PMOR approaches based on interpolation will be investigated and compared with respect to the availability of error bounds and preservation of passivity in the reduced-order model.

\subsection{Interpolation in the frequency domain}

A combination of model reduction of the local systems with interpolation in the frequency domain was first proposed for standard state space systems with $E=I$ in [9] and then re-investigated in [40]. An extension of this method to DAE systems is, however, straightforward [24].

Using the data $\left(p_{j}, \hat{H}_{j}(s)\right)$, where $\hat{H}_{j}(s)=\hat{C}_{j}\left(s \hat{E}_{j}-\hat{A}_{j}\right)^{-1} \hat{B}_{j}$ is the transfer function of the local reduced-order model (6) for $j=0, \ldots, k$, one computes the reduced transfer function $\hat{H}(p, s)$ at any $p \in \mathbb{P}$ by interpolation. Using multivariate polynomial or rational interpolation, we construct the reduced transfer function

$$
\hat{H}(p, s)=\sum_{j=0}^{k} f_{j}(p) \hat{H}_{j}(s),
$$

where the weight functions $f_{j}(p)$ satisfy the conditions $f_{j}\left(p_{i}\right)=\delta_{i j}$ with the Kronecker delta $\delta_{i j}$. It is easy to see that $\hat{H}(p, s)$ fulfills the interpolation conditions $\hat{H}\left(p_{j}, s\right)=\hat{H}_{j}(s)$ for $j=0, \ldots, k$.

In the transient analysis of electrical circuits, it is important to have a state space representation for the reduced transfer function (7). It can be obtained as follows

$$
\hat{H}(p, s)=\sum_{j=0}^{k} f_{j}(p) \hat{H}_{j}(s)=\sum_{j=0}^{k} f_{j}(p) \hat{C}_{j}\left(s \hat{E}_{j}-\hat{A}_{j}\right)^{-1} \hat{B}_{j}=\hat{C}(p)(s \hat{E}-\hat{A})^{-1} \hat{B}
$$

where

$$
\begin{aligned}
& \hat{E}=\operatorname{diag}\left(\hat{E}_{0}, \ldots, \hat{E}_{k}\right), \quad \hat{A}=\operatorname{diag}\left(\hat{A}_{0}, \ldots, \hat{A}_{k}\right), \\
& \hat{B}=\left[\hat{B}_{0}^{T}, \ldots, \hat{B}_{k}^{T}\right]^{T}, \quad \hat{C}(p)=\left[f_{0}(p) \hat{C}_{0}, \ldots, f_{k}(p) \hat{C}_{k}\right] .
\end{aligned}
$$

Since, initially, only the matrices $E$ and $A$ are parameter-dependent, one may prefer to place $f_{j}(p)$ into $\hat{E}$ and $\hat{A}$ by writing $\hat{H}(p, s)=\hat{C}(s \hat{E}(p)-\hat{A}(p))^{-1} \hat{B}$, where

$$
\begin{aligned}
& \hat{E}(p)=\operatorname{diag}\left(\hat{E}_{0} / f_{0}(p), \cdots, \hat{E}_{k} / f_{k}(p)\right), \\
& \hat{A}(p)=\operatorname{diag}\left(\hat{A}_{0} / f_{0}(p), \cdots, \hat{A}_{k} / f_{k}(p)\right), \\
& \hat{B}=\left[\hat{B}_{0}^{T}, \ldots, \hat{B}_{k}^{T}\right]^{T}, \quad \hat{C}=\left[\hat{C}_{0}, \ldots, \hat{C}_{k}\right],
\end{aligned}
$$

provided $f_{j}(p) \neq 0$ for $j=0, \ldots, k$.

If the reduced-order local systems (6) are passive and reciprocal, then the approximate model (4), (8) is also reciprocal independently of the interpolation method used. However, passivity can be guaranteed only if the weight functions 
satisfy the positivity conditions $f_{j}(p) \geq 0$ for $j=0, \ldots, k$. For example, linear Bsplines fulfill these conditions, whereas the Lagrange polynomial or cubic splines do not meet this property. We can also use other positive interpolation schemes considered in [2].

To measure the quality of the approximation, the error between the original and the reduced-order systems should be estimated. To this end, the following norm

$$
\|H\|_{\mathcal{L}_{\infty}(\mathbb{P}) \otimes \mathcal{H}_{\infty}}:=\sup _{p \in \mathbb{P}} \sup _{s \in \mathbb{C}_{+}}\|H(p, s)\|_{2}
$$

will be used, where $\|\cdot\|_{2}$ denotes the spectral matrix norm. In this definition, we require that $\mathbb{P} \subset \mathbb{R}^{d}$ is bounded and closed, $E(p)$ and $A(p)$ depend continuously on $p$, and $H(p, s)$ is proper for all $p \in \mathbb{P}$ and analytic for all $s \in \mathbb{C}_{+}$. Then the approximation error can be estimated as

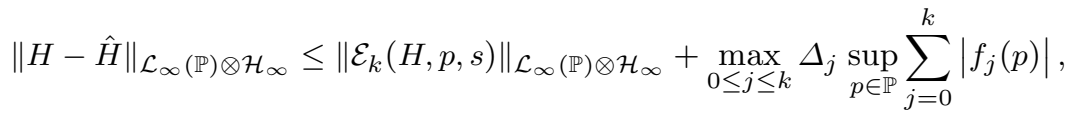

where

$$
\mathcal{E}_{k}(H, p, s)=H(p, s)-\sum_{j=0}^{k} f_{j}(p) \hat{H}_{j}(s)
$$

is the interpolation error, and

$$
\Delta_{j}=\left\|H\left(p_{j}, \cdot\right)-\hat{H}_{j}(\cdot)\right\|_{\mathcal{H}_{\infty}}:=\sup _{s \in \mathbb{C}_{+}}\left\|H\left(p_{j}, s\right)-\hat{H}_{j}(s)\right\|_{2}
$$

is the local $\mathcal{H}_{\infty}$-norm error [9]. Using the linear spline interpolation and assuming that the transfer function $H(p, s)$ of system (2) satisfies the Lipschitz condition

$$
\left\|H\left(p_{1}, \cdot\right)-H\left(p_{2}, \cdot\right)\right\|_{\mathcal{H}_{\infty}} \leq L\left\|p_{1}-p_{2}\right\|
$$

for all $p_{1}, p_{2} \in \mathbb{P}$ and a Lipschitz constant $L>0$, we obtain the following error estimate

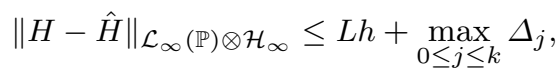

where $\Delta_{j}$ are as in (9), and $h$ is the maximum of the diameters of the subdomains in $\mathbb{P}$ generated by the grid points $p_{0}, \ldots, p_{k}$. Condition (10) can be guaranteed if $E(p)$ and $A(p)$ are both continuously differentiable with respect to $p$ and, additionally, either the transfer function is proper or its polynomial part is independent of $p$. Estimate (11) was proved in [40] for one-dimensional parameter space. An extension for the multi-dimensional case is, however, straightforward. Note that the PABTEC algorithm $[38,39]$ provides computable error bounds for the local systems, that, in turn, implies the existence of the error bounds for the reduced-order parameterized system.

Finally, we want to make some remarks on the dimension of the reduced parameterized system (4) obtained by interpolation in the frequency domain. This system has the state space dimension $r_{0}+\ldots+r_{k}$, where $r_{j}$ is the state space dimension of the local reduced system (6). In practice, not as simple as, e.g., heat equations, quite many circuit systems are not well reducible. Hence, in many cases one will end up with a reduced-order model being of about the same dimension as 
the original one, or even larger than that if the number of interpolation points is large. One remedy for this problem, which has been proposed in [9], is to employ a sparse grid technique [16]. Accordingly, the number of grid points, and, therefore, the overall reduced order can be reduced significantly. Another approach is based on using local interpolation schemes such as linear spline interpolation or local inverse distance weighted interpolation [2], where the dimension of the reduced model is independent of the number of interpolation points and does not exceed $(d+1) r$ for simplicial grids and $2^{d} r$ for rectangular grids with $d$ being the dimension of the parameter domain $\mathbb{P}$ and $r=\max \left\{r_{0}, \ldots, r_{k}\right\}$. Local interpolation schemes can certainly combined with the sparse grid technique.

\subsection{Interpolation on the manifold of projection subspaces}

We would like to begin this subsection by a note that the set of all subspaces in $\mathbb{R}^{N}$ of fixed dimension, say $r$, forms a Riemannian manifold [1,25]. It is called Grassmann manifold and denoted by $\mathfrak{G}(r, N)$. Therefore, the interpolation of projection subspaces is actually performed on this manifold. It was realized in [4] that the interpolation of the matrices whose columns span the corresponding subspaces does not work in general. One reason is that the subspaces spanned by resulted matrices may not belong to the considered manifold. To solve this problem, a 4-step procedure was proposed in [4] where the interpolation process was done on the tangent spaces of the Grassmann manifold.

We will denote by $\mathfrak{T}_{\mathcal{W}_{0}} \mathfrak{G}(r, N)$ the tangent space of $\mathfrak{G}(r, N)$ at $\mathcal{W}_{0}$, by $\log _{\mathcal{W}_{0}}(\mathcal{W})$ the logarithm of $\mathcal{W}$ on the Grassmann manifold, and by $\operatorname{Exp}_{\mathcal{W}_{0}}(\mathcal{Y})$ the exponent of $\mathcal{Y} \in \mathfrak{T}_{\mathcal{W}_{0}} \mathfrak{G}(r, N)$, see [1] for detail. Next, we present a procedure for interpolating the right projection subspaces $\mathcal{W}_{0}, \ldots, \mathcal{W}_{k}$.

Step 1 Choose a contact point for the tangent space, e.g., $\mathcal{W}_{0}$.

Step 2 Map $\mathcal{W}_{1}, \cdots, \mathcal{W}_{k}$ to $\mathfrak{T}_{\mathcal{W}_{0}} \mathfrak{G}(r, N)$ by $\log _{\mathcal{W}_{0}}$. For this purpose, we compute the thin singular value decomposition (SVD)

$$
\left(I-W_{0}\left(W_{0}^{T} W_{0}\right)^{-1} W_{0}^{T}\right) W_{j}\left(W_{0}^{T} W_{j}\right)^{-1}\left(W_{0}^{T} W_{0}\right)^{1 / 2}=U_{W_{j}} \Sigma_{W_{j}} V_{W_{j}}^{T}
$$

for $j=1, \ldots, k$. Then the image $\mathcal{Y}_{W_{j}}=\log _{\mathcal{W}_{0}}\left(\mathcal{W}_{j}\right)$ is spanned by the columns of a matrix

$$
Y_{W_{j}}=U_{W_{j}} \arctan \left(\Sigma_{W_{j}}\right) V_{W_{j}}^{T}, \quad j=1, \ldots k,
$$

and $\mathcal{Y}_{W_{0}}=\log _{\mathcal{W}_{0}}\left(\mathcal{W}_{0}\right)=0$

Step 3 Interpolate on $\mathfrak{T}_{\mathcal{W}_{0}} \mathfrak{G}(r, N)$ using some standard interpolation technique: for a parameter value $p \in \mathbb{P}$, the columns of a matrix

$$
Y_{W}(p)=\sum_{j=1}^{k} f_{j}(p) Y_{W_{j}}
$$

where $f_{j}(p)$ are weights depending on the interpolation method, span the subspace $\mathcal{Y}_{W}(p)$ that belongs to $\mathfrak{T}_{\mathcal{W}_{0}} \mathfrak{G}(r, N)$. 
Step 4 Map $\mathcal{Y}_{W}(p)$ back to the Grassmann manifold $\mathfrak{G}(r, N)$ by $\operatorname{Exp}_{\mathcal{W}_{0}}$. For this purpose, one has to compute first the thin SVD

$$
Y_{W}(p)=U_{W}(p) \Sigma_{W}(p) V_{W}(p)^{T}
$$

and then the matrix representation

$$
W(p)=W_{0}\left(W_{0}^{T} W_{0}\right)^{-1 / 2} V_{W}(p) \cos \left(\Sigma_{W}(p)\right)+U_{W}(p) \sin \left(\Sigma_{W}(p)\right)
$$

of the sought-after subspace $\mathcal{W}(p)=\operatorname{Exp}_{\mathcal{W}_{0}}\left(\mathcal{Y}_{W}(p)\right)$.

A similar procedure is performed to interpolate the left projection subspaces $\mathcal{Z}_{1}, \ldots, \mathcal{Z}_{k}$ to get $\mathcal{Z}(p)$ with the basis matrix

$$
Z(p)=Z_{0}\left(Z_{0}^{T} Z_{0}\right)^{-1 / 2} V_{Z}(p) \cos \left(\Sigma_{Z}(p)\right)+U_{Z}(p) \sin \left(\Sigma_{Z}(p)\right) .
$$

Then the system matrices of the reduced-order model (4) can be determined by projection

$$
\begin{array}{ll}
\hat{E}(p)=Z^{T}(p) E(p) W(p), & \hat{A}(p)=Z^{T}(p) A(p) W(p), \\
\hat{B}(p)=Z^{T}(p) B, & \hat{C}(p)=C W(p) .
\end{array}
$$

The procedure presented above is the same as in [4] except for the formulas (12), (15) and (16) adapted from [3]. The difference comes from the fact that in [4], the bases $W_{j}$ and $Z_{j}$ are required to be orthonormal, i.e, $W_{j}^{T} W_{j}=Z_{j}^{T} Z_{j}=I$ for $j=0, \ldots, k$, while we consider here general basis matrices. Unlike PRIMA and SPRIM based on an Arnoldi procedure [7], the columns of the projected matrices in the PABTEC algorithm are not necessarily orthogonal. The generalization in (12), (15) and (16) helps to get rid of the orthogonalization of the bases.

Note that for a parameter $p$ not belonging to the set $\left\{p_{0}, \ldots, p_{k}\right\}$, we have to do all computations (13)-(17) in order to determine the reduced-order system. Working with parametric systems, this action, in general, has to be repeated many times. The computational speed of this stage is, therefore, of great importance. For a class of affine parameter-dependent systems with $E=I$, it was proposed in [41] to decompose the computation process into an offline stage, which is usually computational expensive, and an online stage, in which the computational complexity is independent of the problem dimension $N$. Next, we present an extension of this approach for the DAE system (2).

Suppose that the system matrices $E(p)$ and $A(p)$ in (2) have the following form

$$
E(p)=\sum_{i=1}^{n_{E}} f_{i}^{E}(p) E_{i}, \quad A(p)=\sum_{i=1}^{n_{A}} f_{i}^{A}(p) A_{i}
$$

where $E_{i}$ and $A_{i}$ are independent of $p$. For the effectiveness of the method presented later on, we assume, moreover, that $n_{E}, n_{A} \ll N$ and the evaluation of $f_{i}^{E}(p)$ and $f_{i}^{A}(p)$ is cheap. Such an affine representation for circuit equations can be obtained as follows. The element matrices $\mathcal{C}(p), \mathcal{L}(p)$ and $\mathcal{G}(p)$ are often diagonal and can be written as

$$
\mathcal{C}(p)=\sum_{i=1}^{n_{\mathcal{C}}} f_{i}^{\mathcal{C}}(p) J_{n_{i}^{\mathcal{C}}}, \quad \mathcal{L}(p)=\sum_{i=1}^{n_{\mathcal{L}}} f_{i}^{\mathcal{L}}(p) J_{n_{i}^{\mathcal{L}}}, \quad \mathcal{G}(p)=\sum_{i=1}^{n_{\mathcal{G}}} f_{i}^{\mathcal{G}}(p) J_{n_{i}^{\mathcal{G}}},
$$


where $J_{n_{i}^{*}}$ denotes a square matrix of appropriate size whose all entries are zeros except that the entries from the $\left(n_{i-1}^{*}+1\right)$-th position to the $n_{i}^{*}$-th position on the diagonal are equal to one, and $n_{0}=0$. Then we have

$$
E(p)=\sum_{i=1}^{n_{\mathcal{C}}} f_{i}^{\mathcal{C}}(p) E_{i}^{\mathcal{C}}+\sum_{i=1}^{n_{\mathcal{L}}} f_{i}^{\mathcal{L}}(p) E_{i}^{\mathcal{L}}, \quad A(p)=\sum_{i=1}^{n_{\mathcal{G}}} f_{i}^{\mathcal{G}}(p) A_{i}^{\mathcal{G}}+A^{\mathcal{L} \mathcal{V}}
$$

where

$$
\begin{aligned}
E_{i}^{\mathcal{C}}=\left[\begin{array}{ccc}
A_{\mathcal{C}} J_{n_{i}^{\mathcal{C}}} A_{\mathcal{C}}^{T} & 0 & 0 \\
0 & 0 & 0 \\
0 & 0 & 0
\end{array}\right], & E_{i}^{\mathcal{L}}=\left[\begin{array}{ccc}
0 & 0 & 0 \\
0 & J_{n_{i}^{\mathcal{L}}} & 0 \\
0 & 0 & 0
\end{array}\right], \\
A_{i}^{\mathcal{G}}=\left[\begin{array}{ccc}
-A_{\mathcal{R}} J_{n_{i}^{\mathcal{G}}} A_{\mathcal{R}}^{T} & 0 & 0 \\
0 & 0 & 0 \\
0 & 0 & 0
\end{array}\right], & A^{\mathcal{L} \mathcal{V}}=\left[\begin{array}{ccc}
0 & -A_{\mathcal{L}} & -A_{\mathcal{V}} \\
A_{\mathcal{L}}^{T} & 0 & 0 \\
A_{\mathcal{V}}^{T} & 0 & 0
\end{array}\right] .
\end{aligned}
$$

Let $P_{W} \in \mathbb{R}^{N \times n_{W}}$ be an orthonormal basis of the intersection of the orthogonal complement of the subspace spanned by the columns of $U_{W_{1}}$ and the subspace spanned by that of $\left[U_{W_{2}}, \ldots, U_{W_{k}}\right]$. It is nothing else but the matrix whose columns are the left singular vectors of $\left(I-U_{W_{1}} U_{W_{1}}^{T}\right)\left[U_{W_{2}}, \ldots, U_{W_{k}}\right]$. Note that the number of columns of $P_{W}$ satisfies $n_{W} \leq(k-1) r$. Consider the matrix

$$
K_{W}(p)=\left[\begin{array}{c}
f_{1}(p) \arctan \left(\Sigma_{W_{1}}\right)+f_{2}(p) U_{W_{1}}^{T} Y_{W_{2}} V_{W_{1}}+\ldots+f_{k}(p) U_{W_{1}}^{T} Y_{W_{k}} V_{W_{1}} \\
f_{2}(p) P_{W}^{T}\left(I-U_{W_{1}} U_{W_{1}}^{T}\right) Y_{W_{2}} V_{W_{1}}+\ldots+f_{k}(p) P_{W}^{T}\left(I-U_{W_{1}} U_{W_{1}}^{T}\right) Y_{W_{k}} V_{W_{1}}
\end{array}\right]
$$

where $f_{i}(p), i=1, \ldots, k$ are the interpolation coefficients in (13). Analogously, we construct the matrix $K_{Z}(p)$ from $P_{Z}, \Sigma_{Z_{1}}, U_{Z_{1}}, V_{Z_{1}}$ and $Y_{Z_{2}}, \ldots, Y_{Z_{k}}$. Let

$$
K_{W}(p)=\Phi_{W}(p) S_{W}(p) \Psi_{W}^{T}(p), \quad K_{Z}(p)=\Phi_{Z}(p) S_{Z}(p) \Psi_{Z}^{T}(p)
$$

be the thin SVDs of $K_{W}(p)$ and $K_{Z}(p)$. Then the reduced system matrices are computed as

$$
\begin{aligned}
& \hat{E}(p)=Z^{T}(p) E(p) W(p) \\
& =\sum_{i=1}^{n_{E}} f_{i}^{E}(p) \cos \left(S_{Z}(p)\right) \Psi_{Z}^{T}(p) V_{Z_{1}}^{T}\left(Z_{0}^{T} Z_{0}\right)^{-\frac{1}{2}} Z_{0}^{T} E_{i} W_{0}\left(W_{0}^{T} W_{0}\right)^{-\frac{1}{2}} V_{W_{1}} \Psi_{W}(p) \cos \left(S_{W}(p)\right) \\
& \quad+\sum_{i=1}^{n_{E}} f_{i}^{E}(p) \cos \left(S_{Z}(p)\right) \Psi_{Z}^{T}(p) V_{Z_{1}}^{T}\left(Z_{0}^{T} Z_{0}\right)^{-\frac{1}{2}} Z_{0}^{T} E_{i}\left[U_{W_{1}}, P_{W}\right] \Phi_{W}(p) \sin \left(S_{W}(p)\right) \\
& \quad+\sum_{i=1}^{n_{E}} f_{i}^{E}(p) \sin \left(S_{Z}(p)\right) \Phi_{Z}^{T}(p)\left[U_{Z_{1}}, P_{Z}\right]^{T} E_{i} W_{0}\left(W_{0}^{T} W_{0}\right)^{-\frac{1}{2}} V_{W_{1}} \Psi_{W}(p) \cos \left(S_{W}(p)\right) \\
& \quad+\sum_{i=1}^{n_{E}} f_{i}^{E}(p) \sin \left(S_{Z}(p)\right) \Phi_{Z}^{T}(p)\left[U_{Z_{1}}, P_{Z}\right]^{T} E_{i}\left[U_{W_{1}}, P_{W}\right] \Phi_{W}(p) \sin \left(S_{W}(p)\right)
\end{aligned}
$$


The matrix $\hat{A}(p)$ is constructed similarly. The input and output matrices are

$$
\begin{aligned}
\hat{B}(p) & =Z^{T}(p) B \\
& =\cos \left(S_{Z}(p)\right) \Psi_{Z}^{T}(p) V_{Z_{1}}^{T}\left(Z_{0}^{T} Z_{0}\right)^{-\frac{1}{2}} Z_{0}^{T} B+\sin \left(S_{Z}(p)\right) \Phi_{Z}^{T}(p)\left[U_{Z_{1}}, P_{Z}\right]^{T} B, \\
\hat{C}(p) & =B^{T} W(p) \\
& =B^{T} W_{0}\left(W_{0}^{T} W_{0}\right)^{-\frac{1}{2}} V_{W_{1}} \Psi_{W}(p) \cos \left(S_{W}(p)\right)+B^{T}\left[U_{W_{1}}, P_{W}\right] \Phi_{W}(p) \sin \left(S_{W}(p)\right),
\end{aligned}
$$

respectively. Then, the offline-online decomposition runs as follows.

Offline: Compute

- $W_{j}$ and $Z_{j}$ corresponding to $p_{j}$ for $j=0, \ldots, k$;

- $\left[U_{W_{j}}, \Sigma_{W_{j}}, V_{W_{j}}\right]$ and $\left[U_{Z_{j}}, \Sigma_{Z_{j}}, V_{Z_{j}}\right]$ representing $\log _{\mathcal{W}_{0}}\left(\mathcal{W}_{j}\right)$ and $\log _{\mathcal{Z}_{0}}\left(\mathcal{Z}_{j}\right)$, respectively, for $j=1, \ldots, k$;

- $P_{W}$ and $P_{Z}$ from the thin SVDs of the matrices $\left(I-U_{W_{1}} U_{W_{1}}^{T}\right)\left[U_{W_{2}}, \ldots, U_{W_{k}}\right]$ and $\left(I-U_{Z_{1}} U_{Z_{1}}^{T}\right)\left[U_{Z_{2}}, \ldots, U_{Z_{k}}\right]$.

Compute and store

- all parameter-independent terms for $K_{W}(p)$ in (18) and $K_{Z}(p)$;

- all parameter-independent terms for the reduced system matrices $\hat{E}, \hat{A}, \hat{B}$ and $\hat{C}$ in $(20)-(22)$.

Online: For any value $p \in \mathbb{P}$, we compute

- $K_{W}(p)$ as in (18) and $K_{Z}(p)$;

- the thin SVDs of $K_{W}(p)$ and $K_{Z}(p)$ as in (19);

- the reduced system matrices as in (20)-(22).

Note that unlike $[4,41]$, where one-sided projection methods were used to compute the local reduced-order systems, the above procedure involves two-sided projection that may be advantageous for general DAE systems. However, for the circuit system (2) with

$$
E(p)=E^{T}(p) \geq 0, \quad A(p)+A^{T}(p) \leq 0, \quad C=B^{T},
$$

we have to restrict ourself to one-sided projection (as it is used in SPRIM and PRIMA for general circuits and in PABTEC for RC and RL circuits) in order to guarantee the preservation of passivity and reciprocity in the reduced-order model. Finally, we remark that the model reduction approach based on the interpolation of the projection subspaces does not provide any error bound even if for the local subsystems, error bounds are available.

\subsection{Interpolation in the time domain}

Interpolation of the reduced-order local systems (5) in the time domain is probably the most simple idea for determining a reduced-order model on the whole parameter domain. We, however, do not perform a naive interpolation of the reduced system matrices $\hat{E}_{j}, \hat{A}_{j}, \hat{B}_{j}$ and $\hat{C}_{j}$. Instead, we follow two adjustment methods proposed in [37] and [5], respectively, combined with the interpolation on suitable matrix manifolds as considered in [18].

It was argued in [37] that summing up two different local reduced systems $\left(\hat{E}_{i}, \hat{A}_{i}, \hat{B}_{i}, \hat{C}_{i}\right)$ and $\left(\hat{E}_{j}, \hat{A}_{j}, \hat{B}_{j}, \hat{C}_{j}\right)$ means that we have to accept that the two 
corresponding local state vectors $\hat{x}_{i}$ and $\hat{x}_{j}$ are identical. In fact, it is false, since $\hat{x}_{i}$ and $\hat{x}_{j}$ are the coordinates of the state vector on different projection subspaces $\mathcal{W}_{i}$ and $\mathcal{W}_{j}$ spanned by $W_{i}$ and $W_{j}$, respectively. This observation leads to the use of a common subspace for all local reduced systems without affecting their input-output behavior. This can be done as follows. Let $R_{W}$ and $R_{Z}$ be the $N \times r$ matrices whose columns are the left singular vectors of the matrices $\left[W_{0}, \ldots, W_{k}\right]$ and $\left[Z_{0}, \ldots, Z_{k}\right]$, respectively, corresponding to the $r$ largest singular values. Then the local reduced-order system (5) is transformed into

$$
\begin{aligned}
M_{j} \hat{E}_{j} T_{j} \dot{\tilde{x}}_{j}(t) & =M_{j} \hat{A}_{j} T_{j} \tilde{x}_{j}(t)+M_{j} \hat{B}_{j} u(t), \\
\hat{y}(t) & =\hat{C}_{j} T_{j} \tilde{x}_{j}(t),
\end{aligned}
$$

with $T_{j}=\left(R_{W}^{T} W_{j}\right)^{-1}$ and $M_{j}=\left(Z_{j}^{T} R_{Z}\right)^{-1}$, and the new reduced system matrices are used as the known data in interpolation to get the reduced system matrices at a given value $p$. Note that our left transformation matrix $M_{j}$ differs from that in [37], where $M_{j}=\left(Z_{j}^{T} R_{W}\right)^{-1}$ was chosen. Since we do not see any reason to use information from the right common projection subspace for adjusting the left local projection matrices, our choice seems to be more appropriate. It should be noted that the transformation matrices $T_{j}$ and $M_{j}$ may be ill-conditioned which leads to poor approximation.

Realizing that the direct interpolation of the local reduced-order systems is inadvisable, another approach was proposed in [5] which is based on minimization (not elimination as mentioned in [5]) of the differences between the local projection matrices. This approach can also be extended to DAE systems. First, one chooses the reference projection matrices, e.g., $W_{0}$ and $Z_{0}$, and solves the following minimization problems

$$
Q_{j}=\underset{Q \in \mathbb{G L}(r)}{\operatorname{argmin}}\left\|W_{j} Q-W_{0}\right\|_{F}^{2}, \quad R_{j}=\underset{R \in \mathbb{G L}(r)}{\operatorname{argmin}}\left\|Z_{j} R-Z_{0}\right\|_{F}^{2}
$$

for $j=1, \ldots, k$, where $\mathbb{G} \mathbb{L}(r)$ denotes the set of $r \times r$ nonsingular matrices. After that, $W_{j}$ and $Z_{j}$ are replaced by $W_{j} Q_{j}$ and $Z_{j} R_{j}$, which span the subspaces $\mathcal{W}_{j}$ and $\mathcal{Z}_{j}$, respectively, but are closest to $W_{0}$ and $Z_{0}$ among all the matrices that span $\mathcal{W}_{j}$ and $\mathcal{Z}_{j}$, respectively. The local reduced system matrices become

$$
\tilde{E}_{j}=R_{j}^{T} \hat{E}_{j} Q_{j}, \quad \tilde{A}_{j}=R_{j}^{T} \hat{A}_{j} Q_{j}, \quad \tilde{B}_{j}=R_{j}^{T} \hat{B}_{j}, \quad \tilde{C}_{j}=\hat{C}_{j} Q_{j} .
$$

If $W_{j}$ and $Z_{j}$ have orthonormal columns, then the search for the transformation matrices $Q_{j}$ and $R_{j}$ is restricted to the set of orthogonal matrices [5]. The solutions of these orthogonal minimization problems are given by

$$
Q_{j}=U_{j 0}^{W}\left(V_{j 0}^{W}\right)^{T}, \quad R_{j}=U_{j 0}^{Z}\left(V_{j 0}^{Z}\right)^{T},
$$

where the orthogonal factors are determined from the SVDs

$$
W_{j}^{T} W_{0}=U_{j 0}^{W} \Sigma_{j 0}^{W}\left(V_{j 0}^{W}\right)^{T}, \quad Z_{j}^{T} Z_{0}=U_{j 0}^{Z} \Sigma_{j 0}^{Z}\left(V_{j 0}^{Z}\right)^{T} .
$$

In the general case, the sought-after matrices can be taken

$$
Q_{j}=\left(W_{j}^{T} W_{j}\right)^{-1} W_{j}^{T} W_{0}, \quad R_{j}=\left(Z_{j}^{T} Z_{j}\right)^{-1} Z_{j}^{T} Z_{0}
$$

Note that if these matrices are ill-conditioned, the orthogonal transformation matrices (26) should be preferred. 
Table 1 Logarithmic and exponential mappings for some matrix manifolds

\begin{tabular}{lcc}
\hline Manifold: & nonsingular & $\begin{array}{c}\text { symmetric } \\
\text { positive definite }\end{array}$ \\
\hline $\operatorname{Exp}_{S}(Y)$ & $\exp (Y) S$ & $S^{1 / 2} \exp (Y) S^{1 / 2}$ \\
$\log _{S}(T)$ & $\log \left(T S^{-1}\right)$ & $\log \left(S^{-1 / 2} T S^{-1 / 2}\right)$ \\
\hline
\end{tabular}

The last step is to interpolate the adjusted system matrices. Since the reduced matrices usually own some special properties such as nonsingularity or symmetric positive definiteness, the direct interpolation, like those on Grassmann manifolds, may not work. For this reason, it was suggested in $[5,18]$ to employ the 4 -step procedure mentioned in Subsection 2.2. The only difference is that we have to use different logarithmic and exponential mappings depending on the properties of the matrices to be interpolated. Table 1 provides the appropriate mappings for different matrix manifolds in which exp and log are the standard matrix exponential and matrix logarithm.

For circuit equations, the system matrices satisfy the conditions (23). In case of one-sided projection, the reduced local system matrices maintain these properties meaning, in particular, that $\tilde{E}_{j}$ and $-\tilde{A}_{j}-\tilde{A}_{j}^{T}$ are symmetric and positive semidefinite. For some special circuits, as for example, RC circuits with current sources, one gets even the local reduced-order systems with positive definite $\tilde{E}_{j}$ and $-\tilde{A}_{j}$. Then the interpolation on the appropriate matrix manifold should be used in order to keep these properties in the interpolated reduced system and, as a consequence, to guarantee the preservation of passivity and reciprocity in this system. However, if $\tilde{E}_{j}$ and $\tilde{A}_{j}$ are singular, we cannot compute the logarithmic mapping as in Table 1. In this case, we perform the direct interpolation

$$
\begin{array}{ll}
\hat{E}(p)=\sum_{j=0}^{k} f_{j}(p) \tilde{E}_{j}, & \hat{A}(p)=\sum_{j=0}^{k} f_{j}(p) \tilde{A}_{j}, \\
\hat{B}(p)=\sum_{j=0}^{k} f_{j}(p) \tilde{B}_{j}, & \hat{C}(p)=\sum_{j=0}^{k} f_{j}(p) \tilde{C}_{j},
\end{array}
$$

where the weight functions satisfy $f_{j}(p) \geq 0$ for $j=0, \ldots, k$. These conditions also ensure the preservation of passivity and reciprocity in the reduced-order parametric system.

In Table 2, we summarize the essential properties of the interpolation-based model reduction methods for parameterized circuit systems considered in this section.

\section{Numerical examples}

In this section, we consider some numerical examples in which the presented interpolation-based methods are utilized for model reduction of parameterized circuit equations.

Example 1 The first example is an one-port RCV circuit described by the MNA system (2), (3) of order $N=5000$. The parametrized capacitance and conductance 
Table 2 Properties of the interpolation-based model reduction methods for parameterized circuit systems

\begin{tabular}{lcccc}
\hline Interpolation & Dimension & Passivity & $\begin{array}{c}\text { Error } \\
\text { bound }\end{array}$ & Remarks \\
\hline \hline time domain & $r$ & yes & no & $\begin{array}{l}\text { one-sided projection; adjustment of } \\
\text { the reduced system matrices is needed; } \\
\text { if no interpolation on matrix manifolds } \\
\text { then } f_{j}(p) \geq 0 ;\end{array}$ \\
\hline $\begin{array}{l}\text { projection } \\
\text { subspaces }\end{array}$ & $r$ & yes & no & $\begin{array}{l}\text { online-offline decomposition for affine } \\
\text { parameter-dependent system matri- } \\
\text { ces; one-sided projection }\end{array}$ \\
\hline $\begin{array}{l}\text { frequency } \\
\text { domain }\end{array}$ & $\begin{array}{l}c r \\
(c \leq+1)\end{array}$ & yes & yes & $\begin{array}{l}f_{j}(p) \geq 0 ; \text { reduced local systems may } \\
\text { have different dimensions; passivity } \\
\text { preserving methods for local systems }\end{array}$ \\
\hline
\end{tabular}

matrices have the form $\mathcal{C}(p)=p^{(1)} I$ and $\mathcal{G}(p)=p^{(2)} I$ with $p=\left[p^{(1)}, p^{(2)}\right]^{T} \in \mathbb{P}$ and $\mathbb{P}=\left[10^{-12}, 50 \cdot 10^{-12}\right] \times[1,45]$. The reduced-order local models were computed on the grid $\mathbb{P}_{\text {loc }}=10^{-12}[1,5,15,30,50] \times[1,5,15,30,45]$. For computing these models, we use a balanced truncation model reduction approach presented in [39] and implemented in a MATLAB toolbox PABTEC [42]. This approach is based on a reformulation of the MNA system (2), (3) with $p=p_{j}$ in the modified loop analysis (MLA) form which has the same transfer function $H_{j}(s)$. Frequency inversion of the MLA system yiels $H_{j}^{\star}(s)=H_{j}(1 / s)$. The Lyapunov-based balanced truncation method is then applied to $H_{j}^{\star}(s)$ followed by frequency inversion of the reduced-order model $\hat{H}_{j}^{\star}(s)$ providing the reduced-order model $\hat{H}_{j}(s)=\hat{H}_{j}^{\star}(1 / s)$, see [39] for details. An advantage of this multi-step approach is that the frequencyinverted MLA system $H_{j}^{\star}(s)$ for RCV circuits is symmetric, and, therefore, only one Lyapunov equation has to be solved resulting in one-sided projection. Furthermore, the reduced-order model $\hat{H}_{j}(s)$ is passive and reciprocal, it is exact at zero, i.e., $\hat{H}_{j}(0)=H_{j}(0)$, and there exists a computable error bound on $\left\|\hat{H}_{j}-H_{j}\right\|_{\mathcal{H}_{\infty}}$. Note that the frequency inversion preserves passivity and reciprocity, and both $H_{j}(s)$ and $H_{j}^{\star}(s)$ have the same $\mathcal{H}_{\infty}$-norm. In order to guarantee passivity in the reduced-order interpolated system $\hat{H}(p, s)$ for any parameter $p$, we first perform interpolation in the frequency domain, time domain or on the Grassmann manifold of the reduced-order frequency-inverted models $\hat{H}_{j}^{\star}(s)$, yielding $\hat{H}_{j}^{\star}(p, s)$, and then determine a realization of $\hat{H}_{j}(p, s)=\hat{H}_{j}^{\star}(p, 1 / s)$.

In our experiments, order of all local reduced models is $r_{j}=26$. To measure the quality of approximations, we use a rough estimate of the relative error

$$
\frac{\max _{\omega \in\left[\omega_{\min }, \omega_{\max }\right]}\|H(p, i \omega)-\hat{H}(p, i \omega)\|_{2}}{\max _{\omega \in\left[\omega_{\min }, \omega_{\max }\right]}\|H(p, i \omega)\|_{2}}
$$

with $\omega_{\min }=10$ and $\omega_{\max }=10^{16}$. In Figure 2a), we present the relative error (27), where the reduced-order transfer function $\hat{H}(p, s)$ is obtained via linear spline interpolation in the frequency domain and has the order $r=104$. Figure $2 \mathrm{~b}$ ) shows 


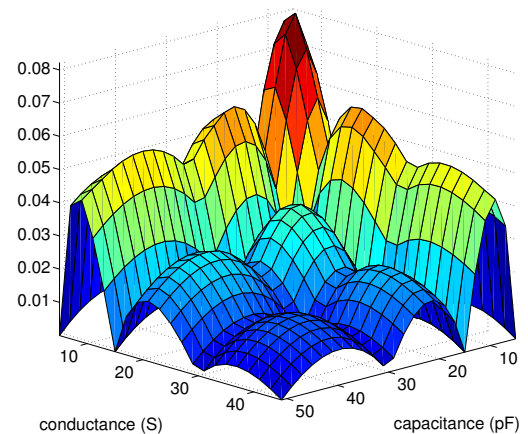

a)

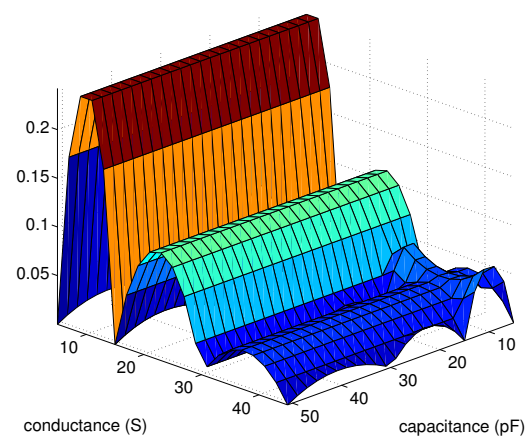

b)

Fig. 2 Example 1: relative errors of linear spline interpolation in the frequency domain (a) and in the time domain (b)

the relative error for the reduced system of order $r=26$ obtained using spline interpolation in the time domain without adjustment. One can see, that the approximation in the first approach is better than that in the second approach. Note that the adjustment with the orthogonal transformation matrices as in (26) does not improve the approximation since these matrices are very close to the identity. Furthermore, the adjusted local systems (24) lead even to a worse approximation.

The relative errors for the approximate models obtained by interpolation on the Grassmann manifold with the reference basis at $p^{(1)}=15 \cdot 10^{-12}$ and $p^{(2)}=15$. are shown in Figure 3a). We observe that the approximation in this approach is much better than that provided by interpolation in the frequency and time domain. The relative error on the whole parameter domain has the same amplitude order as for local reduced models. For comparison, we also present in Figure $3 \mathrm{~b}$ ) the relative errors for interpolation on the Grassmann manifold obtained on a larger parameter domain $\mathbb{P}=\left[10^{-13}, 5.2 \cdot 10^{-10}\right] \times[0.1,460]$ and a coarser grid, where four grid points are just vertices of $\mathbb{P}$. The reference basis is taken at $\left[10^{-13}, 0.1\right]^{T}$. One can see that in this case the approximation quality still remains very good.

Example 2 The second example is the transmission line as presented in Figure 1 with two parameters describing the line length $p^{(1)} \in[10,20]$ measured in mm and the distributed conductance $p^{(2)} \in[0.1,0.9]$ measured in $\mathrm{S} / \mathrm{mm}$. At each point of the grid $[10,12,14,16,18,20] \times[0.1,0.3,0.5,0.7,0.9]$, the original system of order $N=12000$ was approximated by a reduced model of order 27 . Since the original order is rather large, the computation of the relative error on the whole parameter domain is extremely expensive. Instead, we consider the relative error

$$
\frac{\left\|H\left(q_{j}, i \omega\right)-\hat{H}\left(q_{j}, i \omega\right)\right\|_{2}}{\left\|H\left(q_{j}, i \omega\right)\right\|_{2}}
$$

of the frequency response at four points $q_{1}, q_{2}, q_{3}$ and $q_{4}$, henceforth referred to as points of interest, whose locations are depicted in Figure 4. In interpolation of of projection subspaces, we choose the subspace at $p_{0}=[14,0.5]^{T}$ as the contact point for the tangent space. 


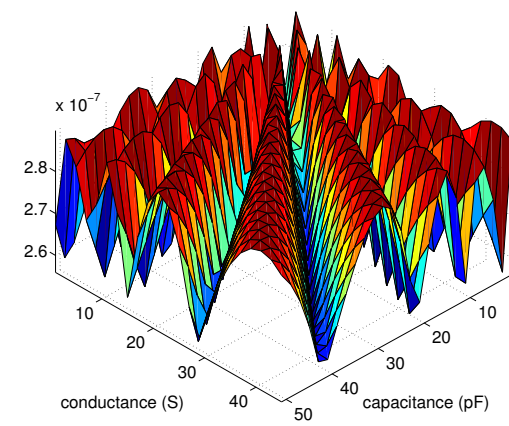

a)

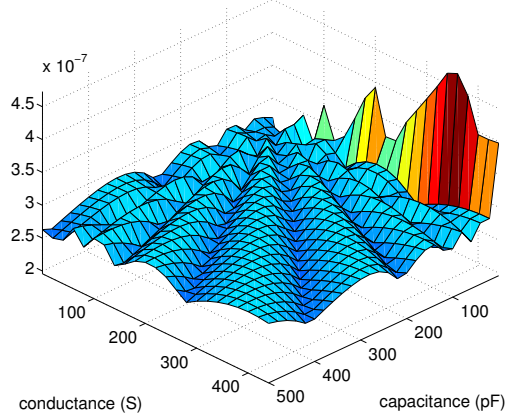

b)

Fig. 3 Example 1: relative errors of linear spline interpolation on the Grassmann manifold using the fine grid $\mathbb{P}_{\text {loc }}(\mathrm{a})$ and the coarse grid $\left[10^{-13}, 5.2 \cdot 10^{-10}\right] \times[0.1,460]$ (b)

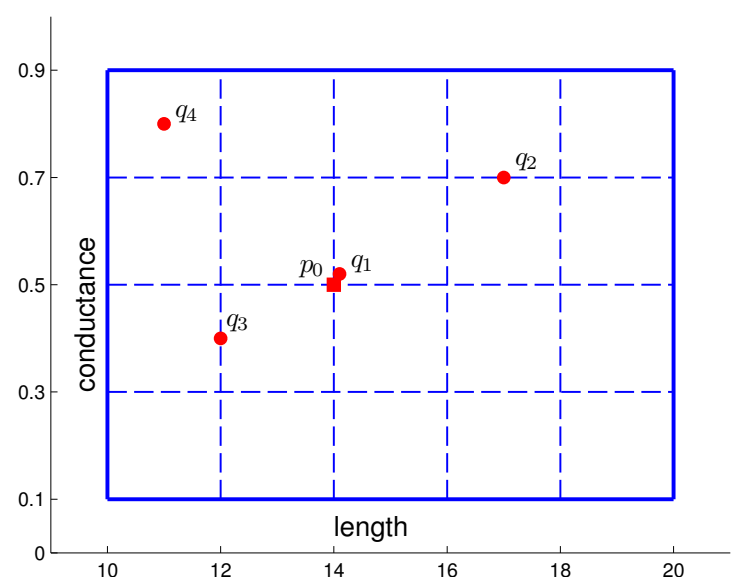

Fig. 4 Example 2: points of interest in the parameter domain

Figures 5a) and 5b) show the relative errors at four points of interest for the reduced-order models determined by spline interpolation in the frequency domain and on the Grassmann manifold, respectively. These approaches provide slightly different results for different points of interest. In Figures 6a) and 6b), we present absolute errors $|y(t)-\hat{y}(t)|$ in the output for both methods at two points $q_{1}$ and $q_{3}$, respectively. The simulation is performed with the input $u(t)=\sin (100 \pi t)^{10} \mathrm{~V}$ on the time interval $[0,0.05]$ with the stepsize $\tau=5 \cdot 10^{-5}$. For comparison, we also include the simulation results for the reduced-order model obtained by interpolation in the frequency domain using the local inverse distance weighted interpolation method. Once again, the approximation by interpolation of projection subspaces is seen to be much better (especially at $q_{3}$ ) than the others. 


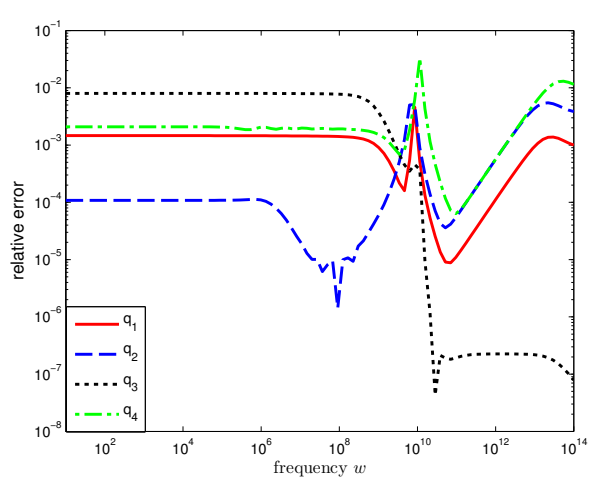

a)

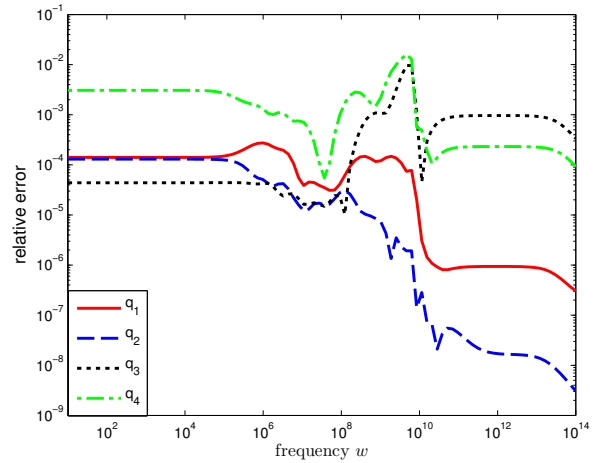

b)

Fig. 5 Example 2: relative errors at four points of interest for linear spline interpolation in the frequency domain (a) and on the Grassmann manifold (b)

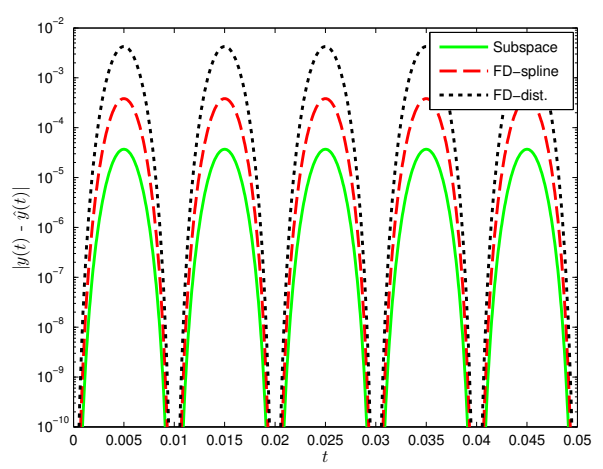

a)

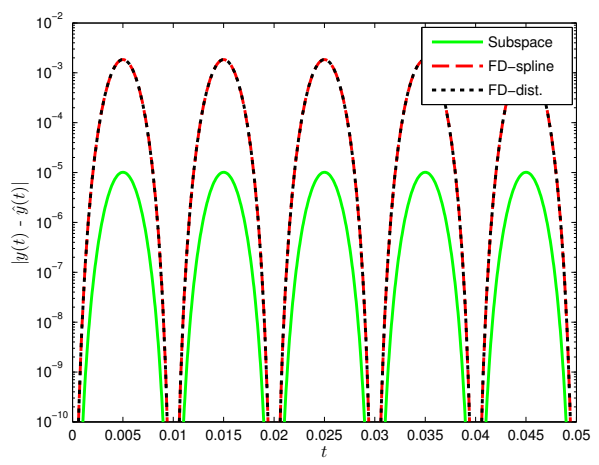

b)

Fig. 6 Example 2: absolute errors in the output produced by interpolation of projection subspaces (Subspace) and interpolation in the frequency domain using linear splines (FDspline) and distance weighted interpolation (FD-dist.) at $q_{1}$ (a) and $q_{3}$ (b)

Some main observations from numerical experiments with presented and also other circuit systems can be summarized as follows.

- Interpolation of projection subspaces yields better approximations than interpolation in the frequency and time domain.

- Within interpolation in the time domain, interpolation of adjusted system matrices as proposed in [5] gives better results than that presented in [37].

- Within interpolation in the frequency domain, the errors caused by linear spline interpolation is smaller than that caused by local distance weighted interpolation.

\section{Conclusion}

In this paper, the problem of model order reduction of parameterized circuit equations was considered. We have presented the recently developed parametric model 
reduction methods based on interpolation in the frequency domain, interpolation of projection subspaces and interpolation in the time domain and discussed their application to circuit systems. Exploiting specific system structure, we have also investigated preservation of passivity and reciprocity in the reduced-order model on the whole parameter domain.

\section{References}

1. Absil, P.A., Mahony, R., Sepulchre, R.: Riemannian geometry of Grassmann manifolds with a view on algorithmic computations. Acta Appl. Math 8, 199-220 (2004)

2. Allasia, G.: Simultaneous interpolation and approximation by a class of multivariate positive operators. NumerAlg 34, 147-158 (2003)

3. Amsallem, D., Cortial, J., Carlberg, K., Farhat, C.: A method for interpolating on manifolds structural dynamics reduced-order models. Internat. J. Numer. Methods Engng $\mathbf{8 0}(9), 1241-1258(2009)$

4. Amsallem, D., Farhat, C.: Interpolation method for adapting reduced-order models and application to aeroelasticity. AIAAJ 46(7), 1803-1813 (2008)

5. Amsallem, D., Farhat, C.: An online method for interpolating linear reduced-order models. SIAM J. Sci. Comput. 33(5), 2169-2198 (2011)

6. Antoulas, A., Beattie, C., Gugercin, S.: Interpolatory model reduction of large-scale dynamical systems. In: J. Mohammadpour, K. Grigoriadis (eds.) Efficient Modeling and Control of Large-Scale Systems, pp. 3-58. Springer-Verlag (2010)

7. Arnoldi, W.: The principle of minimized iterations in the solution of the matrix eigenvalue problem. Quart. Appl. Math. 9, 17-29 (1951)

8. Baur, U., Beattie, C., Benner, P., Gugercin, S.: Interpolatory projection methods for parameterized model reduction. SIAM J. Sci. Comput. 33(5), 2489-2518 (2011)

9. Baur, U., Benner, P.: Modellreduktion fr parametrisierte Systeme durch balanciertes Abschneiden und Interpolation. at-Automatisierungstechnik 57(8), 411-419 (2009)

10. Baur, U., Benner, P., Greiner, A., Korvink, J., Lienemann, J., Moosmann, C.: Parameter preserving model order reduction for mems applications. Math. Comput. Model. Dyn. Syst. 17(5), 297-317 (2011)

11. Benner, P., Breiten, T.: On $\mathcal{H}_{2}$-model reduction of linear parameter-varying systems. In: Proceedings in Applied Mathematics and Mechanics, vol. 11, pp. 805-806 (2011)

12. Benner, P., Feng, L.: A robust algorithm for parametric model order reduction based on implicit moment matching. In: A. Quarteroni, R. Rozza (eds.) Reduced Order Methods for Modeling and Computational Reduction, vol. 9, pp. 159-186. Springer-Verlag, Berlin, Heidelberg (2014)

13. Benner, P., Gugercin, S., Willcox, K.: A survey of model reduction methods for parametric systems. Tech. Rep. MPIMD/13-14, Max Planck Institute, Magdeburg (2013). Available from http://www.mpi-magdeburg.mpg.de/preprints/

14. Bond, B.N., Daniel, L.: A piecewise-linear moment-matching approach to parameterized model-order reduction for highly nonlinear systems. IEEE T. Comput. Aid. D. 26(12), 1467-1480 (2007)

15. Bui-Thanh, T., Willcox, K., Ghattas, O.: Model reduction for large-scale systems with high-dimensional parametric input space. SIAM J. Sci. Comput. 30(6), 3270-3288 (2008)

16. Bungartz, H.J., Griebel, M.: Sparse grids. Acta Numerica 13, 147-269 (2004)

17. Daniel, L., Siong, O., Chay, L., Lee, K., White, J.: A multiparameter moment-matching model-reduction approach for generating geometrically parameterized interconnect performance models. IEEE Trans. Computer-Aided Design Integr. Circuits Syst. 23(5), 678-693 (2004)

18. Degroote, J., Vierendeels, J., Willcox, K.: Interpolation among reduced-order matrices to obtain parameterized models for design, optimization and probabilistic analysis. Int. J. Numer. Meth. Fl. 63, 207-230 (2010)

19. D'Elia, M., Dedé, L., Quarteroni, A.: Reduced basis method for parameterized differential algebraic equations. Bol. Soc. Esp. Math. Apl. 46, 45-73 (2009)

20. Elfadel, I.M., Ling, D.D.: A block rational Arnoldi algorithm for multipoint passive modelorder reduction of multiport RLC networks. In: Digest of Technical Papers of 1997 IEEE/ACM Inter. Conf. Computer-Aided Design, pp. 66-71 (1997) 
21. Farle, O., Hill, V., Ingelström, P., Dyczij-Edlinger, R.: Multi-parameter polynomial order reduction of linear finite element models. Math. Comput. Model. Dyn. Systems 14, 421$434(2008)$

22. Feldmann, P., Freund, R.: Efficient linear circuit analysis by Padé approximation via the Lanczos process. IEEE Trans. Computer-Aided Design Integr. Circuits Syst. 14, 639-649 (1995)

23. Feng, L., Rudnyi, E., Korvink, J.: Preserving the film coefficient as a parameter in the compact thermal model for fast electrothermal simulation. IEEE Trans. Computer-Aided Design Integr. Circuits Syst. 24(12), 1838-1847 (2005)

24. Ferranti, F., Antonini, G., Dhaene, T., Knockaert, L.: Passivity-preserving interpolationbased parameterized model order reduction of peec models based on scattered grids. Int. J. Numer. Model. 24(5), 478-495 (2011)

25. Ferrer, J., García, M.I., Peurta, F.: Differentiable families of subspaces. Linear Algebra Appl. 199, 229-252 (1994)

26. Freund, R.: Krylov-subspace methods for reduced-order modeling in circuit simulation. J. Comput. Appl. Math. 123(1-2), 395-421 (2000)

27. Freund, R.: The sprim algorithm for structure-preserving order reduction of general rcl circuits. model reduction for circuit simulation. In: P. Benner, M. Hinze, E. ter Maten, J. W. (eds.) Model Reduction for Circuit Simulation, Lecture Notes in Electrical Engineering, vol. 74, pp. 25-52. Springer (2011)

28. Gunupudi, P., Khazaka, R., Nakhla, M.: Analysis of transmission line circuits using multidimensional model reduction techniques. IEEE T. Adv. Pack. 25(2), 174-180 (2002)

29. Haasdonk, B., Ohlberger, M.: Efficient reduced models and a posteriori error estimation for parametrized dynamical systems by offline/online decomposition. Math. Comp. Model. Dyn. 17(2), 145-161 (2011)

30. Hay, A., Borggaard, J., Akhtar, I., Pelletier, D.: Reduced-order models for parameter dependent geometries based on shaped sensitivity analysis. J. Comput. Phys. 229, 1327$1353(2010)$

31. J.F. Villena, L.S.: SPARE - A scalable algorithm for passive, structure preserving, parameter-aware model reduction. IEEE Trans. Computer-Aided Design Integr. Circuits Syst. 29(6), 925-938 (2010)

32. Lefteriu, S., Antoulas, A.C., Ionita, A.C.: Parametric model order reduction from measurements. In: The IEEE 19th Conference on Electrical Performance of Electronic Packaging and Systems, pp. 193-196 (2010)

33. Leung, A.T., Khazaka, R.: Parametric model order reduction technique for design optimization. In: IEEE Int. Symp. Circ. Syst. ISCAS 2005, vol. 2, pp. 1290-1293 (2005)

34. Li, Y.T., Bai, Z., Su, Y.: A two-directional Arnoldi process and its application to parametric model order reduction. J. Comput. Appl. Math. 226, 10-21 (2009)

35. Moore, B.: Principal component analysis in linear systems: controllability, observability, and model reduction. IEEE Trans. Automat. Control AC-26(1), 17-32 (1981)

36. Odabasioglu, A., Celik, M., Pileggi, L.: PRIMA: Passive reduced-order interconnect macromodeling algorithm. IEEE Trans. Circuits Syst. 17(8), 645-654 (1998)

37. Panzer, H., Mohring, J., Eid, R., Lohmann, B.: Parametric model order reduction by matrix interpolation. at-Automatisierungtechnik 58(8), 475-484 (2010)

38. Reis, T., Stykel, T.: PABTEC: Passivity-preserving balanced truncation for electrical circuits. IEEE Trans. Computer-Aided Design Integr. Circuits Syst. 29(9), 1354-1367 (2010)

39. Reis, T., Stykel, T.: Lyapunov balancing for passivity-preserving model reduction of RC circuits. SIAM J. Appl. Dyn. Syst. 10(1), 1-34 (2011)

40. Son, N.: Interpolation based parametric model order reduction. Ph.D. thesis, Universität Bremen, Germany (2012)

41. Son, N.: A real time procedure for affinely dependent parametric model order reduction using interpolation on grassmann manifolds. Internat. J. Numer. Methods Engng. 93(8), 818-833 (2013)

42. Steinbrecher, A., Stykel, T.: Element-based model reduction in circuit simulation. In: P. Benner (ed.) System Reduction for Nanoscale IC Design, Mathematics in Industry. Springer-Verlag

43. Stykel, T.: Balancing-related model reduction of circuit equations using topological structure. In: P. Benner, M. Hinze, E. ter Maten (eds.) Model Reduction for Circuit Simulation, Lecture Notes in Electrical Engineering, vol. 74, pp. 53-80. Springer-Verlag, Berlin, Heidelberg (2011)

44. Vlach, J., Singhal, K.: Computer Methods for Circuit Analysis and Design. Kluwer Academic Publisherd, Dordrecht (2003) 